\title{
Fast and direct measurement of the linear birefringence profile in standard single-mode optical fibers
}

\author{
Luis Costa,,${ }^{1, *}$ (1) Regina Magalhães, ${ }^{1}$ (i) Luca Palmieri, ${ }^{2}$ Hugo Martins, ${ }^{3}$ (]) \\ Sonia Martin-Lopez, ${ }^{1}$ María R. Fernández-Ruiz, ${ }^{1}$ and Miguel Gonzalez-Herraez ${ }^{1}$ \\ ${ }^{1}$ Department of Electronics, University of Alcalá, Alcalá de Henares, 28805, Madrid, Spain \\ ${ }^{2}$ Department of Information Engineering, University of Padova, Via G. Gradenigo 6/B, 35131 Padova, Italy \\ ${ }^{3}$ Instituto de Óptica, CSIC, 28006 Madrid, Spain \\ *Corresponding author: luis.duarte@uah.es
}

Received 8 November 2019; revised 19 December 2019; accepted 21 December 2019; posted 23 December 2019 (Doc. ID 382559); published 22 January 2020

Phase birefringence in optical fibers typically fluctuates over their length due to geometrical imperfections induced from the drawing process or during installation. Currently commercially available fibers exhibit remarkably low birefringence, prompting a high standard for characterization methods. In this work, we detail a method that uses chirped-pulse phase-sensitive optical time-domain reflectometry to directly measure position-resolved linear birefringence of single-mode optical fibers. The technique is suitable for fiber characterization over lengths of tens of kilometers, relying on a fast measurement $(\sim 1$ s) with single-ended access to the fiber. The proposed method is experimentally validated with three different commercial single-mode optical fibers. () 2020 Optical Society of America

https://doi.org/10.1364/OL.382559

Provided under the terms of the OSA Open Access Publishing Agreement Ideally, single-mode optical fibers consist of circularly symmetric waveguides with isotropic material properties and no preferential orientation for the electric field. Nonetheless, imperfections in the geometry arising during the drawing process, and elasto-optic effects induced during cabling/installation (notably fiber bending [1]), induce local anisotropies and asymmetries that manifest as birefringence. This translates into polarization mode dispersion (PMD), which is a limiting factor for long-haul, high-bit-rate connections in modern communication links. The problems of PMD in optical fibers have been recognized since the genesis of fiber optic communication technology [2], and consequently, great strides have been made in methods to quantify and mitigate the fiber birefringence. While some strategies focused on reducing the cumulative effects of PMD without notably altering the strength of the birefringence vector [3], manufacturing techniques and characterization methods developed hand in hand, leading to commercial fibers with extremely low birefringence.

Distributed characterization of birefringence is achieved by either techniques relying on indirect estimation or those based on direct measurements. Indirect techniques, such as Brillouin optical time domain reflectometry (BOTDR) [4] or polarization optical time-domain reflectometry (P-OTDR) [5-7], infer local or average birefringence through mathematical models, by studying the evolution of the backscattered polarization state and relating the beat length of two orthogonally polarized waves to the medium birefringence. Direct techniques, on the other hand, are capable of position-resolved measurements of the fiber birefringence. These implementations rely on either optical frequency domain reflectometry (OFDR) [8,9], dynamic Brillouin gratings (DBGs) $[10,11]$, or phase-sensitive $(\phi)$ OTDR [12]. OFDR offers extremely high spatial resolutions, on the order of millimeters, but is limited to the characterization of short fiber lengths. Direct DBG measurements, on the other hand, demand strict polarization alignment and frequency tuning of the three interacting waves (two counter-propagating pump waves and the probe wave that interrogates the DBG), and need access to both ends of the fiber. Also, DBG-based methods have reported only on the characterization of high birefringence fibers $[10,11]$. Lastly, the $\phi$ OTDR measurements exhibited to date require a time-consuming sweep and averaging process, and have been demonstrated on older single-mode optical fibers, having measured birefringence one order of magnitude above what is present in the current state of the art [12].

Techniques based on $\phi$ OTDR measure changes in the optical path across a section of fiber spanning half the probe pulse width, by measuring the interference resulting from light that is elastically backscattered by intrinsic inhomogeneities within the fiber (hereby referred to as scatterers). Essentially, any such section of fiber functionally corresponds to an interferometer of densely packed, low reflectivity mirrors. Thus, any change induced to the optical path may be offset (and thus quantified) by a corresponding frequency shift. Most commonly, the frequency shift is estimated by cross-correlating several consecutive acquisitions of the backscattered power trace, over the course of a time-consuming, wide-band frequency sweep of the probe light [13]. However, a recent variant, chirped-pulse $\phi$ OTDR (CP- $\phi$ OTDR) [14] enables fast probing of a relatively wide band of frequencies in a single shot. This is accomplished by 
mapping each frequency detuning to a specific time section of the pulse, so any locally induced change to the optical path may then be estimated as local time delay in the recovered backscattered power trace. This technique has been successfully used to quantify several parameters, most notably dynamic strain, with remarkable performances $[15,16]$, over long distances with fast sampling rates.

In this work, we propose a technique based on a slight alteration of traditional CP- $\phi$ OTDR aimed at fiber local birefringence characterization. In particular, the presented method directly recovers the birefringence spatial profile of state-of-theart single-mode optical fibers with high sensitivity, over long lengths, and in a short measurement time $(\sim 1 s)$.

It has been previously shown that in a backscattering-based estimation process, such as the one employed in this work, any circular birefringence is indistinguishable from a rotation of the linear birefringence vector [17]. Essentially, the round-trip phase difference between any given pair of scatterers $i, j$, in a linearly birefringent medium, may be expressed as

$$
\Delta \phi_{i j}^{s, f}=\frac{4 \pi L_{i j}}{\lambda}(\bar{n} \pm B / 2)
$$

for linear polarized light along the two axes of linear birefringence (slow, $\phi_{i j}^{s}$, corresponding to the choice of “+”, and fast, $\phi_{i j}^{f}$, to the choice of “-”), where $B$ is the birefringence of the medium, $\bar{n}$ is the average index for unpolarized light, and $L_{i j}$ is the distance between scatterers. On the other hand, for the case of circular birefringence, when sending either right or left circularly polarized light, we expect the following:

$$
\begin{aligned}
\Delta \phi_{i j}^{s, f} & =\frac{2 \pi L_{i j}}{\lambda}\left(\bar{n} \pm B_{\circlearrowright} / 2\right)+\frac{2 \pi L_{i j}}{\lambda}\left(\bar{n} \mp B_{\circlearrowright} / 2\right) \\
& =\frac{4 \pi L_{i j}}{\lambda} \bar{n},
\end{aligned}
$$

where, for circular birefringence $B_{\circlearrowright}$, we see the phase difference measured between any two given scatterers is in principle cancelled by virtue of the round trip. Hence, only the linear birefringence component may affect the CP- $\phi$ OTDR measurement.

In order to now outline our method of estimation of the linear birefringence of the medium, we describe any given homogeneous section of the optical fiber by its two orthogonal eigenstates of polarization $\left(\hat{v}_{1}\right.$ and $\hat{v}_{2}$, such that $\left.\hat{v}_{1}^{*} \cdot \hat{v}_{2}=0\right)$ and the corresponding effective indices. Any polarization $\hat{s}$ may be decomposed into those eigenstates as $\hat{s}=\left(\hat{v}_{1}^{*} \cdot \hat{s}\right) \hat{v}_{1}+\left(\hat{v}_{2}^{*} \cdot \hat{s}\right) \hat{v}_{2}$. In general, any state of polarization of light, $\hat{s}$, rotates unpredictably (albeit deterministically) during propagation within a single-mode fiber. The electric field backscattered at any given fiber section may then be generally described as

$$
e(t)=\left(\hat{v}_{1}^{*} \cdot \hat{s}\right) e_{1}(t) \hat{v}_{1}+\left(\hat{v}_{2}^{*} \cdot \hat{s}\right) e_{2}(t) \hat{v}_{2} .
$$

Hence, the photodetected CP- $\phi$ OTDR signal equals

$$
q(t)=\left|\hat{v}_{1} \cdot \hat{s}\right|^{2} p(t-\tau)+\left|\hat{v}_{2} \cdot \hat{s}\right|^{2} p(t+\tau),
$$

where $p(t)$ is the recovered CP- $\phi$ OTDR power trace for an effective index $\bar{n}$, and $\tau$ corresponds to the chirp-induced delay for a $\Delta n=B / 2$, as given by the system's sensitivity [14]:

$$
\tau=-\frac{v_{0}}{\delta v} \frac{t_{p}}{\bar{n}}\left(\frac{B}{2}\right),
$$

with $v_{0}$ being the laser frequency, $\delta v$ the chirp bandwidth, and $t_{p}$ the pulse width. It is now convenient to represent polarization by $3 \mathrm{D}$ unit Stokes vectors. Let $\hat{V}$ be the Stokes vector associated with $\hat{v}_{1}$, so $-\hat{V}$ is the one associated with $\hat{v}_{2}$. Having $\hat{S}$ be the Stokes vector of $\hat{s}$, we can write

$$
\left|\hat{v}_{i} \cdot \hat{s}\right|^{2}=\frac{1}{2}(1 \pm \hat{V} \cdot \hat{S})=\frac{1}{2}(1 \pm \gamma),
$$

where $\gamma=\hat{V} \cdot \hat{S}$, and $i=1,2$ (corresponding to the choice of the "+" or "-" case, respectively). For polarization $\hat{S}$ or its orthogonal state $-\hat{S}$, the retrieved signals are linear combinations of the signals recovered from the two polarization eigenstates of the fiber:

$$
\begin{aligned}
& q_{1}(t)=\frac{1}{2}[(1+\gamma) p(t-\tau)+(1-\gamma) p(t+\tau)], \\
& q_{2}(t)=\frac{1}{2}[(1-\gamma) p(t-\tau)+(1+\gamma) p(t+\tau)] .
\end{aligned}
$$

Cross-correlating the two components leads to

$$
\begin{aligned}
R_{12}(t)= & \frac{1}{4}\left[2\left(1-\gamma^{2}\right) c(t)+(1+\gamma)^{2} c(t-2 \tau)\right. \\
& \left.+(1-\gamma)^{2} c(t+2 \tau)\right],
\end{aligned}
$$

where $c(t)$ is the autocorrelation of $p(t)$. Notice that whenever light is aligned with the eigenstates of polarization of the fiber $(\gamma=1)$, Eq. (7) simplifies to $q_{1}(t)=p(t-\tau)$ and $q_{2}(t)=p(t+\tau)$, and Eq. (8) simplifies to $R_{12}(t)=c(t-2 \tau)$, as expected.

A common estimator of time delay employed in CP- $\phi$ OTDR is the generalized cross-correlation (GCC) algorithm [15], i.e., finding the lag at which there is a maximum of cross correlation,

$$
\delta=\operatorname{argmax}\left\{R_{12}(t)\right\} .
$$

To calculate the result of this estimator when applied to Eq. (8), we describe the peak of the correlation function $c(t)$ as a Gaussian peak; so let $c(t)=\exp \left[-t^{2} /\left(2 w^{2}\right)\right]$. The position $\delta$ of the cross-correlation peak is determined by setting $d R_{12} / d t=0$. Note that the maximum peak shift is obtained when the signal polarization is aligned with one of the two eigenstates, in which case, the peak shift is exactly $\pm 2 \tau$; therefore, in general, $|\delta| \leq 2 \tau$. Using this constraint, the peak position is well approximated by

$$
\delta \approx 2 \gamma \tau,
$$

where the approximation holds as long as $w^{2} \gg \tau^{2}$. From a practical point of view, this means that Eq. (10) has a fairly good validity as long as the birefringence-induced delay $\tau$ is smaller than the width $w$ of the correlation peak. This width is inversely proportional to the chirp bandwidth $(w \approx 1 / \delta v)$; so by Eq. $(5)$, we see that the condition $\tau<w$ is equivalent to $B<2 \bar{n} /\left(v_{0} t_{p}\right)$. For a pulse of a few meters, this corresponds to a limit of the measurable birefringence on the order of $10^{-6}$, confirming the method can be applied to standard telecommunication fibers.

The process to obtain a local estimation of birefringence consists of sending light with the pairs of polarization states 


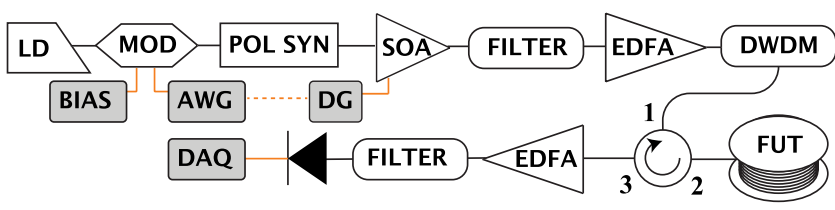

Fig. 1. Schematics of the optical setup. LD, laser driver; MOD, amplitude modulator; POL SYN, polarization synthesizer; FILTER, tunable filter; BIAS, bias controller; AWG, arbitrary waveform generator; DG, delay generator; EDFA, erbium-doped fiber amplifier; DWDM, dense wavelength division multiplexer; FUT, fiber under test; DAQ, oscilloscope.

$0^{\circ} / 90^{\circ},+45^{\circ} /-45^{\circ}$ and left/right circular [i.e., with Stokes vectors $\hat{S} \pm(1,0,0), \pm(0,1,0)$, and $\pm(0,0,1)]$ into the fiber. These will evolve unpredictably during propagation but remain orthogonal among themselves, thus forming an orthogonal basis of the Poincaré sphere. Measuring $\delta$ for the three pairs yields the projection of $\hat{V}$ in this basis. It follows that

$$
\sqrt{\delta_{1}^{2}+\delta_{2}^{2}+\delta_{3}^{2}}=2 \tau \sqrt{\hat{V}_{1}^{2}+\hat{V}_{2}^{2}+\hat{V}_{3}^{2}}=2 \tau,
$$

where $2 \tau$ is the time delay that corresponds to the section's linear birefringence, $B$ [Eq. (5)], and $\hat{V}_{i}$ is the projection of $\hat{V}$ on the local basis vector $\hat{S}_{i}$.

In the case of circular or elliptical local birefringence, we must consider the decomposition of the state into linear and circular components, where only the former gives a nonzero contribution to the measured delay, as stated in Eq. (2).

The assembled CP- $\phi$ OTDR (Fig. 1) consists of an external cavity laser in continuous emission, externally modulated with a chirped, pulsed waveform [generated by an arbitrary waveform generator (AWG)], with the modulator work point set to minimum transmission of the carrier. The resulting signal is sent through a polarization synthesizer and time-gated through a semiconductor optical amplifier (SOA). Any remaining carrier and the generated lower sideband are extinguished through a tunable filter, set to allow only the upper sideband within the filter passband.

The pulse is amplified by an erbium-doped fiber amplifier (EDFA), followed by a $100 \mathrm{GHz}$ dense wavelength division multiplexer (DWDM) to mitigate some of the amplified spontaneous emission (ASE), and sent into the fiber under test through an optical circulator. The recovered light is then amplified, filtered, and photodetected. We employ a lowphase-noise laser $(<0.1 \mathrm{kHz})$, thus avoiding the need for phase-noise compensation [18] and a $20 \mathrm{GHz}$ photodetector, well above the chirp bandwidth of $4 \mathrm{GHz}$. The digital acquisition is done at $10 \mathrm{GS} / \mathrm{s}$, and the laser is pulsed with $1 \mathrm{kHz}$ repetition rate and $50 \mathrm{~ns}$ of pulse width. Each crosscorrelation is re-sampled to 10 times higher sampling through sinc interpolation, followed by a parabolic fit of the main peak [15].

For the demonstration, three single-mode G.652D fiber spools from different manufacturers (of lengths 1,4 , and $10 \mathrm{~km}$ ) are used. The polarization synthesizer is programmed to cycle through the six orthogonal polarization states $[(1,0,0) ;(0,1,0)$; $(0,0,1) ;(-1,0,0) ;(0,-1,0) ;(0,0,-1)]$, holding each state for $300 \mathrm{~ms}$ in the two shorter fibers (1.8 s measurement) and $150 \mathrm{~ms}$ for the $10 \mathrm{~km}$ fiber $(0.9 \mathrm{~s}$ measurement), due to memory

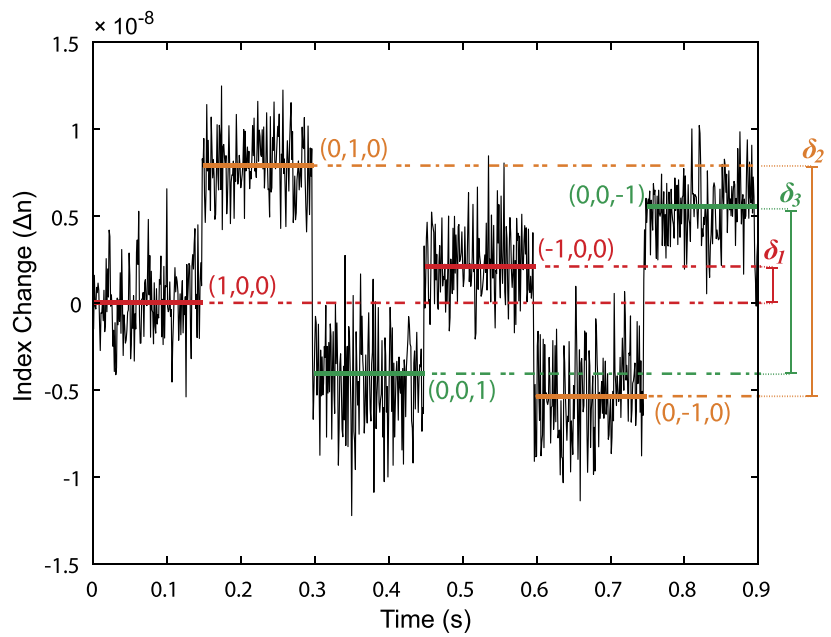

Fig. 2. Example acquisition for a given position in the fiber. The values of $\delta$ required in Eq. (11) are obtained as the perceived index difference between each orthogonal pair $(\hat{S},-\hat{S})$. The numbers in the figure represent the $\hat{S}$ vector of light at the input for that given time section.

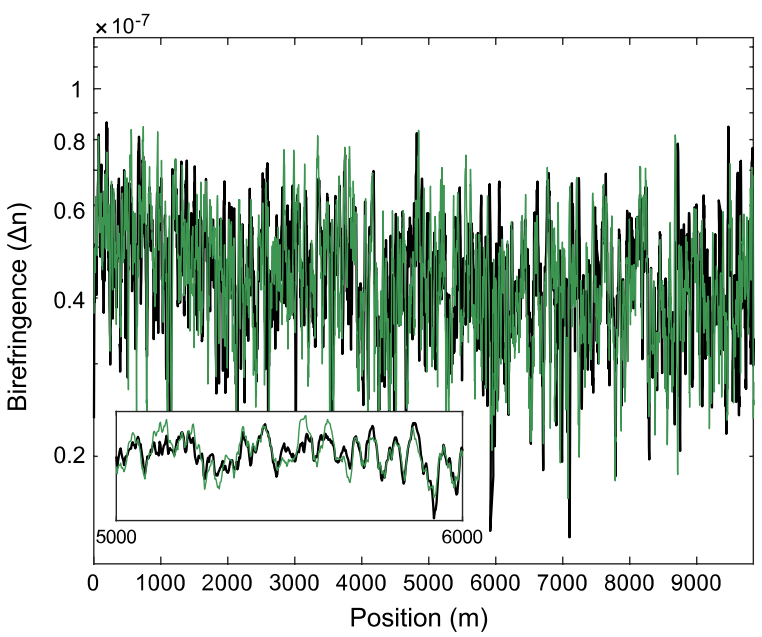

Fig. 3. Birefringence profile obtained from both ends for the $10 \mathrm{~km}$ fiber. Spatial resolution of $25 \mathrm{~m}$.

constraints of the acquisition device. The time series acquired for any interrogated position of the fiber consists of a noisy, piecewise-constant function. Each constant time section is then averaged to estimate the effective index shift for that given polarization state. The value of $\delta_{p}$ at position $z$ is then measured as the total index change between orthogonal pairs. An example of the measurement procedure is depicted in Fig. 2.

The self-consistency of the method is then tested by comparing the birefringence spatial profiles, as obtained from each end of the same fiber. Figure 3 shows the obtained results for the $10 \mathrm{~km}$ fiber. For denoising and better visualization of the results, the measured birefringence is smoothed with a moving mean filter over $25 \mathrm{~m}$. Figure 4 shows the same experiment in three independent cases: the concatenation of the $4 \mathrm{~km}$ and $1 \mathrm{~km}$ fibers, the $4 \mathrm{~km}$ fiber, and the $1 \mathrm{~km}$ fiber. Though some small decorrelation between each corresponding measured pair is expected to appear due to measurement noise and changes in the 


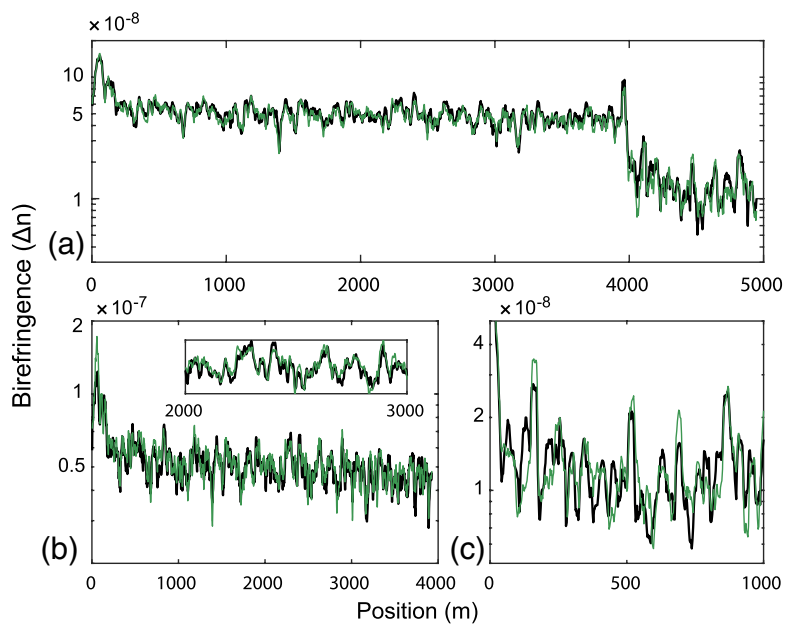

Fig. 4. Birefringence profile obtained from both ends of (a) $4 \mathrm{~km}+$ $1 \mathrm{~km}$ fiber concatenated; (b) $4 \mathrm{~km}$ fiber; (c) $1 \mathrm{~km}$ fiber. Spatial resolution of $25 \mathrm{~m}$.

temperature gradient of the room over the course of each acquisition, the correlation coefficient between both is notably high, despite the explicitly low amount of birefringence of the fibers under test. These results are summarized in Table 1 (see column "Corr. Coeff."), along with the mean birefringence of each fiber $[$ mean $(B)]$ and the standard deviation of the difference between measurements from both extremes of the fiber (as depicted in Figs. 3 and 4$), \sigma_{12}$.

The technique displays the ability to measure levels of birefringence intrinsic to the manufacturing process in state-ofthe-art single-mode fibers. Moreover, the noticeable decrease in the average birefringence level on the longer fibers $(\approx 20 \%$ in the $10 \mathrm{~km}$ fiber and $\approx 10 \%$ in the $4 \mathrm{~km}$ fiber) is consistent with the effect of spooling. Actually, lower values of $z$ in Figs. 3 and 4 correspond to the inner layers of the spool, where the bending radius is smaller, and hence the bending-induced birefringence [1] is higher. As the measurement proceeds to the outer layers, this deterministic contribution to birefringence decreases in intensity. The measured variation of average birefringence agrees with a radius variation of some millimeters.

To conclude, we propose a method to quickly retrieve spatially resolved measurements of remarkably low linear birefringence in long optical fiber links, with single-ended access to the fiber.

Though this work is restricted to fiber lengths up to $10 \mathrm{~km}$, this limit is imposed by the acquisition hardware memory and not from the technique itself. We expect the actual limits to match the current state of the art for a CP- $\phi$ OTDR (on the order of several tens of kilometers [19]).

The spatial resolution may also be tuned for the application, up to the limit imposed by half the pulse width $(5 \mathrm{~m}$, in our experiments). Whenever the birefringence vector undergoes fast spatial changes (e.g., with spun fibers), the measurement at any given position consists of the magnitude of the effective linear birefringence over the measured spatial resolution [20].

All experimental measurements consisted of a fast acquisition of a single cycle of the six polarization states (totaling 1 to $2 \mathrm{~s}$ ), so we expect that performance could be further improved by averaging several consecutive cycles, in applications where speed is not a major concern. Note that, since the underlying model
Table 1. Summarized Results ${ }^{a}$

\begin{tabular}{lccc}
\hline FUT & $\boldsymbol{m e a n}(\boldsymbol{B})$ & $\boldsymbol{\sigma}_{\mathbf{1 2}}$ & Corr. Coeff. \\
\hline $1 \mathrm{~km}$ & $1.4 \mathrm{e}-8$ & $3.1 \mathrm{e}-9$ & 0.91 \\
$4 \mathrm{~km}$ & $5.3 \mathrm{e}-8$ & $4.1 \mathrm{e}-9$ & 0.92 \\
$10 \mathrm{~km}$ & $4.5 \mathrm{e}-8$ & $7.2 \mathrm{e}-9$ & 0.81 \\
\hline
\end{tabular}

${ }^{a}$ Descriptions for each result in the main text.

assumes small time delays of the optical power trace, further work and analysis are required to assess the suitability of this method for highly birefringent fibers.

This work may pave the road for fast polarization-based distributed sensing in standard single-mode fibers [21], as well as complete characterization of fiber links, when used in combination with other techniques such as P-OTDR.

Funding. Ministerio de Economía y Competitividad (TEC 2015-71127-C2-2-R); Ministerio de Ciencia, Innovación y Universidades (FJCI-2016-27881, IJCI-2017-33856, RTI 2018-097957-B-C31); Comunidad de Madrid (SINFO TON2-CM:P2018/NMT-4326); European Commission (PCIN-2015-219 DOMINO, MSCA-ITN-ETN-722509).

Disclosures. The authors declare no conflicts of interest.

\section{REFERENCES}

1. L. Palmieri, A. Galtarossa, and T. Geisler, Opt. Lett. 35, 2481 (2010).

2. I. P. Kaminow, IEEE J. Quantum Electron. 17, 15 (1981).

3. L. Palmieri, T. Geisler, and A. Galtarossa, Opt. Express 20, 1 (2012).

4. Y. Lu, X. Bao, L. Chen, S. Xie, and M. Pang, Opt. Lett. 37, 3936 (2012).

5. A. J. Rogers, Appl. Opt. 20, 1060 (1981).

6. J. N. Ross, Appl. Opt. 21, 3489 (1982).

7. A. Galtarossa and L. Palmieri, J. Lightwave Technol. 22, 1103 (2004).

8. M. Wegmuller, M. Legré, and N. Gisin, J. Lightwave Technol. 20, 828 (2002).

9. M. E. Froggatt, D. K. Gifford, S. Kreger, M. Wolfe, and B. J. Soller, J. Lightwave Technol. 24, 4149 (2006).

10. M. A. Soto, A. Denisov, X. Angulo-Vinuesa, S. Martin-Lopez, L. Thévenaz, and M. Gonzalez-Herraez, Proc. SPIE. 10323, $103238 Z$ (2017).

11. Y. Dong, L. Teng, H. Zhang, T. Jiang, and D. Zhou, Characterization of Distributed Birefringence in Optical Fibers (Springer, 2018), pp. 1-31.

12. M. A. Soto, X. Lu, H. F. Martins, M. Gonzalez-Herraez, and L. Thévenaz, Opt. Express 23, 24923 (2015).

13. Y. Koyamada, M. Imahama, K. Kubota, and K. Hogari, J. Lightwave Technol. 27, 1142 (2009).

14. J. Pastor-Graells, H. F. Martins, A. Garcia-Ruiz, S. Martin-Lopez, and M. Gonzalez-Herraez, Opt. Express 24, 13121 (2016).

15. L. Costa, H. F. Martins, S. Martín-López, M. R. Fernández-Ruiz, and M. González-Herráez, J. Lightwave Technol. 37, 4487 (2019).

16. H. D. Bhatta, L. Costa, A. Garcia-Ruiz, M. R. Fernandez-Ruiz, H. F. Martins, M. Tur, and M. Gonzalez-Herraez, J. Lightwave Technol. 37, 4888 (2019).

17. A. Galtarossa, D. Grosso, L. Palmieri, and L. Schenato, Opt. Lett. 33, 2284 (2008).

18. M. R. Fernández-Ruiz, J. Pastor-Graells, H. F. Martins, A. GarciaRuiz, S. Martin-Lopez, and M. Gonzalez-Herraez, J. Lightwave Technol. 36, 979 (2018).

19. J. Pastor-Graells, J. Nuño, M. R. Fernández-Ruiz, A. Garcia-Ruiz, H. F. Martins, S. Martin-Lopez, and M. Gonzalez-Herraez, J. Lightwave Technol. 35, 4677 (2017).

20. L. Palmieri, J. Lightwave Technol. 24, 4075 (2006).

21. A. Galtarossa and L. Palmieri, Proc. SPIE 10323, 207 (2017). 